\title{
STRONG RATIONAL CONNECTEDNESS OF TORIC VARIETIES
}

\author{
Yifei Chen And Vyacheslav Shokurov
}

\begin{abstract}
In this paper, we establish that, for any given finitely many distinct points $P_{1}, \ldots, P_{r}$ and a closed subvariety $S$ of codimension $\geq 2$ in a complete toric variety $X$ over an algebraically closed field of characteristic 0 , there exists a rational curve $f: \mathbb{P}^{1} \rightarrow X$ passing through $P_{1}, \ldots, P_{r}$, disjoint from $S \backslash\left\{P_{1}, \ldots, P_{r}\right\}$ (see Main Theorem). As a corollary we obtain that the smooth loci of complete toric varieties are strongly rationally connected.
\end{abstract}

\section{Contents}

1. Introduction

2. Preliminaries

3. Proof of main theorem

\section{Introduction}

The concept of rationally connected varieties was independently invented by KollárMiyaoka-Mori [1] and Campana [2]. This class of variety has interesting arithmetic and geometric properties.

A class of proper rationally connected varieties comes from the smooth Fano varieties [2], [3] or [4]. Shokurov [5], Zhang [6], Hacon and $\mathrm{M}^{\mathrm{c}}$ Kernan [7] proved that Fano type (FT) varieties are rationally connected.

An interesting question is whether the smooth locus of a rationally connected variety is rationally connected. In general the answer of the question is NO (For example, see [8], Example 19). However, for the FT (or log del Pezzo) surface case, Keel and $\mathrm{M}^{\mathrm{c}}$ Kernan gave an affirmative answer, that is, if $(S, \Delta)$ is a log del Pezzo surface, then its smooth locus $S^{\mathrm{sm}}$ is rationally connected [9], but this does not imply the strong rational connectedness.

The concept of strongly rationally connected varieties (see Definition 2.2) was first introduced by Hassett and Tschinkel [8]. A proper and smooth separably rationally connected variety $X$ over an algebraically closed field is strongly rationally connected (see [1] 2.1 or [4] IV.3.9). Xu [10] proved that the smooth loci of log del Pezzo surfaces are not only rationally connected but also strongly rationally connected, which confirms a conjecture of Hassett and Tschinkel [8], Conjecture 20). It is expected that

\footnotetext{
Received by the editors June 7, 2010.
} 
the smooth locus of an FT variety is strongly rationally connected (cf. Example 2.1 and Main Theorem).

Throughout the paper, we work over an algebraically closed field of characteristic 0 . It is interesting whether the Main Theorem holds for any algebraically closed field.

Main Theorem. Let $X$ be a complete toric variety over an algebraically closed field of characteristic 0 . Let $p_{1}, \ldots, p_{r} \in \mathbb{P}^{1}$ be $r$ distinct points. Then, for any given distinct points $P_{1}, \ldots, P_{r} \in X$ ( $P_{i}$ possibly singular), there is a geometrically free rational curve $f: \mathbb{P}^{1} \rightarrow X$ over $\left\{P_{i}\right\}, 1 \leq i \leq r$ (see Definition 2.4 and Remark 2.2) with $f\left(p_{i}\right)=P_{i}, 1 \leq i \leq r$. Moreover, $f$ can be chosen to be free over $\left\{P_{i}\right\}$ if all points $P_{i}$ are smooth.

Main Theorem implies the following:

Let $X$ be a complete toric variety over an algebraically closed field of characteristic 0 . Let $p_{1}, \ldots, p_{r} \in \mathbb{P}^{1}$ be $r$ distinct points. Then, for any given distinct points $P_{1}, \ldots, P_{r} \in X$ (possibly singular) and any given codimension $\geq 2$ subvariety $S \subseteq X$, there is a rational curve $f: \mathbb{P}^{1} \rightarrow X$ passing through $P_{1}, \ldots, P_{r}$ with $f\left(p_{i}\right)=P_{i}$, $1 \leq i \leq r$, disjoint from $S \backslash\left\{P_{1}, \ldots, P_{r}\right\}$.

If all points $P_{i} \in X$ are smooth, then we get the following corollary.

Corollary 1.1. The smooth locus of a complete toric variety is strongly rationally connected.

Note that in the Main Theorem, if $\operatorname{dim} X \geq 2$ the curve $f$ can be chosen to be birational (see the proof of Main Theorem). For $\operatorname{dim} X=1$ and any $r$, the curve $f$ can be chosen to be a finite morphism. For $\operatorname{dim} X=0, r \leq 1$ and the curve $f$ is a constant morphism.

In the paper, we suppose that $\operatorname{dim} X \geq 1$ and a rational curve is always a nonconstant morphism $f: \mathbb{P}^{1} \rightarrow X$.

\section{Preliminaries}

When we say that $x$ is a point of a variety $X$, we mean that $x$ is a closed point in $X$.

A normal projective variety $X$ is called FT (Fano Type) if there exists an effective $\mathbb{Q}$-divisor $D$, such that $(X, D)$ is klt and $-\left(K_{X}+D\right)$ is ample. See [11] LemmaDefinition 2.6 for other equivalent definitions.

Let $N \cong \mathbb{Z}^{n}$ be a lattice of rank $n$. A toric variety $X(\Delta)$ is associated to a fan $\Delta$, a finite collection of rational convex cones $\sigma \subset N_{\mathbb{R}}:=N \otimes_{\mathbb{Z}} \mathbb{R}$ (see [12] or [13].

Example 2.1. Projective toric varieties are FT. Let $K$ be the canonical divisor of the projective toric variety $X(\Delta), T$ be the torus of $X$, and $\Sigma=X \backslash T=\sum D_{i}$ be the complement of $T$ in $X$. Then $K$ is linearly equivalent to $-\Sigma$. Since $X$ is projective, there is an ample invariant divisor $L$. Suppose that $L=\sum d_{i} D_{i}$. Let the polytope $\square_{L}=\left\{m \in M \mid\left\langle m, e_{i}\right\rangle+d_{i} \geq 0, \forall e_{i} \in \Delta(1)\right\}$, where $M$ is the dual lattice of $N$, and $\Delta(1)$ is the set consisting of one-dimensional cones in $\Delta$. Let $u$ be an element in the interior of $\square_{L}$. Let $\chi^{u}$ be the corresponding rational function of $u \in M$ (see [12] Section 1.3), and div $\chi^{u}$ be the divisor of $\chi^{u}$. Then $D=\operatorname{div} \chi^{u}+L$ is effective and ample and has support $\Sigma$. That is, $D=\sum d_{i}^{\prime} D_{i}$ and all $d_{i}^{\prime}>0$.

Let $\epsilon$ be a positive rational number, such that all coefficients of prime divisors in $\epsilon D$ are strictly less than 1 . Then $\Sigma-\epsilon D$ is effective. It is well known that $(X, \Sigma-\epsilon D)$ is klt (see, e.g. [14] Lemma 5.2), and $-(K+\Sigma-\epsilon D) \sim \epsilon D$ is ample. Hence, $X$ is FT. 
Definition 2.1. An isogeny of toric varieties is a finite surjective toric morphism. Toric varieties $X$ and $Y$ are said to be isogenous if there exists an isogeny $X \rightarrow Y$. The isogeny class of a toric variety $X$ is a set consisting of all toric varieties $Y$ such that $X$ and $Y$ are isogenous.

Theorem 2.1. Let $f: X \rightarrow Y$ be a finite surjective toric morphism. Then there exists a finite surjective toric morphism $g: Y \rightarrow X$.

Proof. Let $f: X \rightarrow Y$ be a finite surjective toric morphism of toric varieties and $\varphi:\left(N^{\prime}, \Delta^{\prime}\right) \rightarrow(N, \Delta)$ be the corresponding map of lattices and fans. Then we can identify $N^{\prime}$ as a sublattice of $N$ and $\Delta^{\prime}=\Delta$.

There is an positive integer $r$ such that $r N$ is a sublattice of $N^{\prime}$. Let $g$ be the corresponding toric morphism of $(r N, \Delta) \rightarrow\left(N^{\prime}, \Delta\right)$. Since $(r N, \Delta)$ and $(N, \Delta)$ induce an isomorphic toric variety, we get $g: Y \rightarrow X$ is a finite surjective toric morphism.

The properties of isogeny:

(1) Isogeny is an equivalence relation.

(2) If a toric variety $Y$ is in the isogeny class of $X$ and $\mu: X \rightarrow Y$ is the isogeny, then there is a one-to-one correspondence between the set of orbits $\left\{O_{i}^{X}\right\}$ of $X$ and the set of orbits $\left\{O_{i}^{Y}=\mu\left(O_{i}^{X}\right)\right\}$ of $Y$. Hence $\operatorname{dim} O_{i}^{X}=\operatorname{dim} O_{i}^{Y}$ for all $i$, and the number of orbits is independent of the choice of toric varieties in an isogeny class of $X$.

A variety $X$ over a characteristic 0 field is rationally connected, if there is a bounded family of rational curves in $X$, such that any two general points $x_{1}, x_{2} \in X$ can be connected by rational curves in this given family.

Definition 2.2. ([8] Definition 14.) A smooth rationally connected variety $Y$ is strongly rationally connected if any of the following conditions hold:

(1) for each point $y \in Y$, there exists a rational curve $f: \mathbb{P}^{1} \rightarrow Y$ joining $y$ and a generic point in $Y$;

(2) for each point $y \in Y$, there exists a very free rational curve containing $y$;

(3) for any finite collection of points $y_{1}, \ldots, y_{m} \in Y$, there exists a very free rational curve containing the $y_{j}$ as smooth points;

(4) for any finite collection of jets

$$
\text { Spec } k[\epsilon] /\left\langle\epsilon^{N+1}\right\rangle \subset Y, \quad i=1, \ldots, m
$$

supported at distinct points $y_{1}, \ldots, y_{m}$, there exists a very free rational curve smooth at $y_{1}, \ldots, y_{m}$ and containing the prescribed jets.

Definition 2.3. Let $X$ be a complete normal variety, $B$ be a set of finitely many closed points in $\mathbb{P}^{1}$, and $g: B \rightarrow X$ be a morphism. A rational curve $f: \mathbb{P}^{1} \rightarrow X$ is called weakly free over $g$ if there exists an irreducible family of rational curves $T$ and an evaluation morphism ev: $\mathbb{P}^{1} \times T \rightarrow X$ such that

(1) $f=f_{t_{0}}=\left.\mathrm{ev}\right|_{\mathbb{P}^{1} \times t_{0}}$ for some $t_{0} \in T$,

(2) for any $t \in T, f_{t}=\left.\mathrm{ev}\right|_{\mathbb{P}^{1} \times t}$ is a rational curve and $\left.f_{t}\right|_{B}=g$,

(3) the evaluation morphism ev: $\mathbb{P}^{1} \times T \rightarrow X$ by ev $(x, t)=f_{t}(x)$ is dominant.

We say that a rational curve $f^{\prime}: \mathbb{P}^{1} \rightarrow X$ is a general deformation of $f$, or $f^{\prime}$ is a sufficiently general weakly free rational curve, if $f^{\prime}=f_{t}$ is a general member of the family. 
We say that a weakly free rational curve $h: \mathbb{P}^{1} \rightarrow X$ is a general deformation of $f$, if there is a weakly free rational curve $f^{\prime}: \mathbb{P}^{1} \rightarrow X$, with family $T^{\prime}$ and the evaluation morphism ev' $: \mathbb{P}^{1} \times T^{\prime} \rightarrow X$, such that $T^{\prime}$ contains the generic point $\eta$ of $T$ (that is, a scheme point of $T^{\prime}$ isomorphic to the generic point of $T$ ) with $f_{\eta}^{\prime}=\left.\mathrm{ev}^{\prime}\right|_{\mathbb{P}^{1} \times \eta}=f_{\eta}$, and $h=f_{\eta^{\prime}}^{\prime}=\left.\mathrm{ev}^{\prime}\right|_{\mathbb{P}^{1} \times \eta^{\prime}}$, where $\eta^{\prime}$ is the general point of $T^{\prime}$.

Definition 2.4. Let $X$ be a complete normal variety, $B$ be a set of finitely many closed points in $\mathbb{P}^{1}$, and $g: B \rightarrow X$ be a morphism. A rational curve $f: \mathbb{P}^{1} \rightarrow X$ is called geometrically free over $g$ if there exists an irreducible family of rational curves $T$ and an evaluation morphism ev: $\mathbb{P}^{1} \times T \rightarrow X$ such that

(1) $f=f_{t_{0}}=\left.\mathrm{ev}\right|_{\mathbb{P}^{1} \times t_{0}}$ for some $t_{0} \in T$;

(2) for any $t \in T, f_{t}=\left.\mathrm{ev}\right|_{\mathbb{P}^{1} \times t}$ is a rational curve and $\left.f_{t}\right|_{B}=g$;

(3) for any codimension 2 subvariety $Z$ in $X, f_{t}\left(\mathbb{P}^{1}\right) \cap Z \subseteq g(B)$ for general $t \in T$ (general meaning $t$ belongs to a dense open subset in $T$, depending on $Z$ ).

Remark 2.1. If $X$ is smooth projective over an algebraically closed field of characteristic 0 , then weak freeness over $g$ is equivalent to usual freeness over $g$ if $|B| \leq 2$. Indeed, let $f_{t}$ be such a curve that for some point $p \in \mathbb{P}^{1} \backslash B$, the tangent map $d_{(p, t)}: T_{(p, t)} \mathbb{P}^{1} \times T \rightarrow T_{f_{t}(p)} X$ is surjective. Then the sheaf $f_{t}^{*} T_{X} \otimes I_{B}$ is free at $p$, that is, generated by global sections near $p$, where $I_{B}$ is the ideal sheaf of $B$. On the other hand, $f_{t}^{*} T_{X} \otimes I_{B}=\sum \mathcal{O}_{\mathbb{P}^{1}}\left(a_{i}\right)$. Thus by the local generatedness all $a_{i} \geq 0$, $H^{1}\left(P^{1}, f_{t}^{*} T_{X} \otimes I_{B}\right)=0$ and $f_{t}^{*} T_{X} \otimes I_{B}$ is generated by global sections. So $f_{t}$ is free over $g$.

Remark 2.2. In our application, we usually assume that $g$ is one-to-one, and $B=$ $\left\{p_{i}\right\}$ a fixed subset of $\mathbb{P}^{1}$. Let $P_{i}=g\left(p_{i}\right)$. Without confusion, we say that $f$ is geometrically free over $\left\{P_{i}\right\}$ (resp. weakly free over $\left\{P_{i}\right\}$ ) instead of saying that $f$ is geometrically free over $g$ (resp. weakly free over $g$ ).

Weak freeness and geometric freeness are generalizations of usual freeness (see [4] II.3.1 Definition) if the curve passes through singularities. To consider weakly free rational curves or geometrically free rational curves, we think of them as general members in a certain family. In particular, we can suppose that the morphism ev is flat.

Example 2.2. Let $X$ be a projective cone over a conic. Let $T$ be the family of all lines through the vertex $O$. Then $l \in T$ is weakly free and geometrically free over $O$ by the construction.

For a complete normal algebraic variety, geometrical freeness implies weakly freeness (see [15] Proposition 3.3.3). But there exists weakly free rational curves that are not geometrically free. For example, a general rational curve on the projective rational surface in Example 19 [8] is weakly free but not geometrically free.

Let $X$ be a variety and $\pi: \tilde{X} \rightarrow X$ be a resolution, $C \subset \tilde{X}$ be a curve, and $D \subset \tilde{X}$ be a proper subvariety. Let $P_{1}, \ldots, P_{r} \in X$ be $r$ distinct points. We say that the 
curve $C$ intersects $D$ over $\left\{P_{i}\right\}$ only in divisorial points of $D$, if

(1) $\pi^{-1}\left(P_{i}\right) \subset D$ is a divisor for each $i$;

(2) the curve $C$ intersects $D$ properly, and each point of $C \cap \pi^{-1}\left(P_{i}\right)$ lies in a unique irreducible component of $\pi^{-1}\left(P_{i}\right)$ for each $i$. Note that $C$ is possibly singular.

We need the following resolution in the proof of the Main Lemma and the Main Theorem.

Theorem 2.2. Let $X$ be a toric variety. Let $\Sigma$ be the invariant locus of $X$. Let $P_{1}, \ldots, P_{r} \in X$ be $r$ distinct points. Let $f: \mathbb{P}^{1} \rightarrow X$ be a sufficiently general weakly free rational curve over $P_{1}, \ldots, P_{r}$. Then there exists a resolution $\pi: \tilde{X} \rightarrow X$, such that

(1) $\pi^{-1}\left(\Sigma \cup\left\{P_{i}\right\}\right)$ is a divisor with simple normal crossing;

(2) $\pi^{-1}\left(P_{j}\right) \subseteq \pi^{-1}\left(\Sigma \cup\left\{P_{i}\right\}\right)$ is a divisor for each point $P_{j}$;

(3) $\pi: \tilde{X} \rightarrow X$ is an isomorphism over $X \backslash\left(\right.$ Sing $\left.X \cup\left\{P_{i}\right\}\right)$;

(4) sufficiently general $\tilde{f}\left(\mathbb{P}^{1}\right)$ intersects $\pi^{-1}\left(\Sigma \cup\left\{P_{i}\right\}\right)$ over each $P_{i}$ only in divisorial points of $\pi^{-1}\left(\Sigma \cup\left\{P_{i}\right\}\right)$.

More generally, let $f_{j}: \mathbb{P}^{1} \rightarrow X, 1 \leq j \leq m$ be finitely many sufficiently general weakly free rational curve over a subset of $\left\{P_{i}\right\}$, where $\left\{P_{i}\right\}$ is a set of finitely many distinct points in $X$. Then there exists a resolution $\pi: \tilde{X} \rightarrow X$ such that

$\left(1^{\prime}\right) \pi^{-1}\left(\Sigma \cup\left\{P_{i}\right\}\right)$ is a divisor with simple normal crossing;

$\left(2^{\prime}\right) \pi^{-1}\left(P_{i}\right) \subseteq \pi^{-1}\left(\Sigma \cup\left\{P_{i}\right\}\right)$ is a divisor for each point $P_{i}$;

$\left(3^{\prime}\right) \pi: \tilde{X} \rightarrow X$ is an isomorphism over $X \backslash\left(\right.$ Sing $\left.X \cup\left\{P_{i}\right\}\right)$;

$\left(4^{\prime}\right)$ For each $j$, sufficiently general $\tilde{f}_{j}\left(\mathbb{P}^{1}\right)$ intersects $\pi^{-1}\left(\Sigma \cup\left\{P_{i}\right\}\right)$ over each $P_{i}$ only in divisorial points of $\pi^{-1}\left(\Sigma \cup\left\{P_{i}\right\}\right)$, where $\tilde{f}_{j}: \mathbb{P}^{1} \rightarrow \tilde{X}$ is the proper birational transformation of a general deformation of $f_{j}$ and is a (weakly) free rational curve.

Proof. When the ground field is of characteristic 0, (1)-(3) follow from usual facts in the resolution theory, e.g., see [16] Main Theorems I and II. However, in the toric or toroidal case, the same result holds for any field. More precisely, if all $P_{i}$ are invariant, we can use a toric resolution. If some $P_{i}$ are not invariant, they can be converted into toroidal invariant points $P_{i}$ after a toroidalization (for a reference, see Propositoin 3.2 in [17]).

To fulfill (4), we need extra resolution over intersections of the divisorial components of $\pi^{-1}\left(\Sigma \cup\left\{P_{i}\right\}\right)$ through which general $\tilde{f}$ is passing over $P_{i}$. Termination of such resolution follows from an estimate by the multiplicities of intersection for $f\left(\mathbb{P}^{1}\right)$ with $\Sigma$. The last resolution is independent of the choice of a general rational curve by Lemma 3.1 below. However it depends on the choice of intersections of divisorial components. For more details, see the proof of Lemma 4.3.4 in [15].

For the general statement, we can get $\left(1^{\prime}\right)-\left(3^{\prime}\right)$ in a similar manner as above. To fulfil $\left(4^{\prime}\right)$, we just need extra resolutions over each point $P_{i}$.

We discuss some examples of rational curves on projective spaces and the quotients of projective spaces. 
Example 2.3. For any given subvariety $S$ of codimension $\geq 2$ in $\mathbb{P}^{n}$, any points $P_{1}, \ldots, P_{r} \in \mathbb{P}^{n}$, and any integer $d \geq r$, there exists a rational curve $C$ of degree $d$, such that each $P_{i} \in C$ and $C \cap S=\emptyset$.

Indeed, we can construct a tree $T$ with $r$ branches, such that each $P_{i}$ is a smooth points on a unique branch and disjoint from $S$. The tree can be smoothed into a rational curve $C$ passing through $P_{1}, \ldots, P_{r}$, disjoint from $S$. The rational curve $C$ has degree $r$. For $d \geq r$, we can attach $d-r$ rational curves to the tree $T$, and smooth it.

Applying Example 2.3, we get

Example 2.4. Let $\pi: \mathbb{P}^{n} \rightarrow X$ be a finite morphism, $S$ be a codimension $\geq 2$ subvariety in $X$, and $\left\{P_{i}\right\}_{i=1}^{m}$ be a set of $m$ points outside $S$. Then there exists a rational curve $C$, such that each $P_{i} \in C$ and $C \cap S=\emptyset$.

In particular, the same result holds if $X$ is a quotient space $\mathbb{P}^{n} / G$, where $G$ is a finite group, for example, if $X$ is a weighted projective space. It is well known that if $X$ is a complete $\mathbb{Q}$-factorial toric variety with Picard number one, then there exists a weighted projective space $Y$ and a finite toric morphism $\pi: Y \rightarrow X$. So the same result holds for rational curves on a complete $\mathbb{Q}$-factorial toric variety with Picard number one. It is a very special case of our Main Theorem.

\section{Proof of main theorem}

In this section we prove Main Theorem. Let us first prove Main Lemma, which is a special case of Main Theorem.

Main Lemma. Let $X$ be a complete toric variety. Let $P, Q \in X$ be two distinct points ( $P, Q$ possibly singular). Let $S \subseteq X$ be a closed subvariety of codimension $\geq 2$. Then there exists a weakly free rational curve on $X$ over $P, Q$, disjoint from $S \backslash\{P, Q\}$.

To prove Main Lemma, we need some preliminaries.

Lemma 3.1. Let $f$ be a weakly free rational curve on $X$, and $F_{1}, \ldots, F_{s} \subseteq X$ be $s$ proper irreducible subvarieties in $X$. Then there exist $s^{\prime}, 0 \leq s^{\prime} \leq s$, subvarieties among $\left\{F_{j}\right\}$ (after renumbering we assume that they are $F_{1}, \ldots, F_{s^{\prime}}$ ) such that a general deformation of $f$ intersects $F_{1}, \ldots, F_{s^{\prime}}$, and is disjoint from $F_{s^{\prime}+1}, \ldots, F_{s}$.

The proof of this Lemma is a standard exercise in incidence relations. See [15] Lemma 4.3.2 for a detailed proof.

Lemma 3.2. Let $X$ be a complete toric variety. Let $P, Q \in X$ be two points (possibly singular), and $S$ be a closed subvariety of codimension $\geq 2$. Let $F_{1}, \ldots, F_{s}$ be all the irreducible components of Sing $X$. Let $f: \mathbb{P}^{1} \rightarrow X$ be a sufficiently general weakly free rational curve over $P, Q$. Suppose $f\left(\mathbb{P}^{1}\right)$ intersects $F_{1} \backslash\{P, Q\}, \ldots, F_{s^{\prime}} \backslash\{P, Q\}$, and is disjoint from $F_{s^{\prime}+1} \backslash\{P, Q\}, \ldots, F_{s} \backslash\{P, Q\}$. Then there exists a weakly free rational curve $f^{\prime}$ over $\{P, Q\}$, such that $f^{\prime}\left(\mathbb{P}^{1}\right)$ is disjoint from $\left((S \backslash\right.$ Sing $\left.X) \cup F_{s^{\prime}+1} \cup \cdots \cup F_{s}\right) \backslash$ $\{P, Q\}$. Moreover, for any fixed closed subvariety $Z$ of $X$, if $f\left(\mathbb{P}^{1}\right) \cap(Z \backslash\{P, Q\})=\emptyset$, then $f^{\prime}\left(\mathbb{P}^{1}\right) \cap(Z \backslash\{P, Q\})=\emptyset$. 
Proof. Applying Theorem 2.2 to the toric variety $X$ and two points $\{P, Q\}$, we get a resolution $\pi: \tilde{X} \rightarrow X$ satisfying 1)-3) in the theorem and a weakly free rational curve $\tilde{f}: \mathbb{P}^{1} \rightarrow \tilde{X}$ satisfying 4 ) in the theorem. A general deformation $\tilde{f}^{\prime}$ of $\tilde{f}$ is weakly free, so $\tilde{f}^{\prime}$ is free by Remark 2.1 above. Moreover, we can assume that $\tilde{f}^{\prime}$ is disjoint from $(S \backslash \operatorname{Sing} X) \backslash \pi^{-1}\{P, Q\}$ by [4] II.3.7.

On the other hand, let $\Sigma$ be the invariant locus of $X$. Notice that Sing $X \subseteq \Sigma$. Then by Theorem 2.2, $\tilde{f}\left(\mathbb{P}^{1}\right)$ intersects $\pi^{-1}(\Sigma \cup\{P, Q\})$ divisorially over $P, Q$, and $\tilde{f}\left(\mathbb{P}^{1}\right)$ is disjoint from the closure of $\pi^{-1}\left(F_{s^{\prime}+1} \backslash\{P, Q\}\right), \ldots, \pi^{-1}\left(F_{s} \backslash\{P, Q\}\right)$. So the general deformation $\tilde{f}^{\prime}$ of $\tilde{f}$ intersects open subsets of divisors $\pi^{-1}(P)$ and $\pi^{-1}(Q)$, disjoint from the closure of $\left((S \backslash\right.$ Sing $\left.X) \backslash \pi^{-1}\{P, Q\}\right) \cup \pi^{-1}\left(F_{s^{\prime}+1} \backslash\{P, Q\}\right) \cup \cdots \cup$ $\pi^{-1}\left(F_{s} \backslash\{P, Q\}\right)$. We apply Lemma 3.3 by replacing $f^{\prime}$ by $\tilde{f}^{\prime}$, dominant morphism $\mu$ by $\pi: \tilde{X} \rightarrow X,\left\{P_{i}\right\}$ by $\{P, Q\}$, and $S$ by $(S \backslash \operatorname{Sing} X) \cup F_{s^{\prime}+1} \cup \cdots \cup F_{s}$. Then we get the weakly free rational curve $f^{\prime}=\pi \tilde{f}^{\prime}: \mathbb{P}^{1} \rightarrow X$ as a general deformation of $f$ (see Definition 2.3), passing through points $P, Q$ and disjoint from $\left((S \backslash \operatorname{Sing} X) \cup F_{s^{\prime}+1} \cup \cdots\right.$ $\left.\cup F_{s}\right) \backslash\{P, Q\}$.

Moreover, we can assume that $f^{\prime}$ is a weakly free rational curve over $P, Q$, by a base change of the family to which $f^{\prime}$ belongs (For details, see the proof of Lemma 4.3.1 in [15]).

The last statement can be proved similarly.

Lemma 3.3. Let $X, X^{\prime}$ be two complete varieties with $\operatorname{dim} X>0$. Let $\mu: X^{\prime} \rightarrow X$ be a dominant morphism. Then the image of a weakly free rational curve on $X^{\prime}$ is weakly free on $X$ in the following sense:

Let $P_{1}, P_{2}, \ldots, P_{r} \in \mu(X)$ be $r$ distinct points, and $S \subseteq X$ be a closed subvariety. Let $S^{\prime}=\mu^{-1} S$, and $P_{1}^{\prime}, P_{2}^{\prime}, \ldots, P_{r}^{\prime} \in X^{\prime}$ be points such that $\mu\left(P_{i}^{\prime}\right)=P_{i}$ for $i=$ $1, \ldots, r$. If $f^{\prime}: \mathbb{P}^{1} \rightarrow X^{\prime}$ is a weakly free rational curve over $P_{1}^{\prime}, P_{2}^{\prime}, \ldots, P_{r}^{\prime}$, disjoint from $S^{\prime} \backslash\left\{P_{1}^{\prime}, P_{2}^{\prime}, \ldots, P_{r}^{\prime}\right\}$, then $f=\mu \circ f^{\prime \prime}$ is a weakly free rational curve on $X$ over $P_{1}, P_{2}, \ldots, P_{r}$, disjoint from $S \backslash\left\{P_{1}, P_{2}, \ldots, P_{r}\right\}$, where $f^{\prime \prime}$ is a general deformation of $f^{\prime}$.

Proof. Since $f^{\prime}$ is weakly free, ev: $\mathbb{P}^{1} \times T^{\prime} \rightarrow X^{\prime}$ is dominant, where $T^{\prime}$ is the family associated to $f^{\prime}$. Since $\mu: X^{\prime} \rightarrow X$ is dominant, ev: $\mathbb{P}^{1} \times T^{\prime} \rightarrow X^{\prime} \rightarrow X$ is dominant. Hence for general deformation $f^{\prime \prime} \in T^{\prime}$ of $f^{\prime}, f=\mu \circ f^{\prime \prime}$ is a weakly free rational curve on $X$.

Lemma 3.4. Let $X$ be a $\mathbb{Q}$-factorial toric variety, and $O$ be a singular orbit of $X$. Then there exists an isogeny $\mu: Y \rightarrow X$, such that $Y$ is smooth along $\mu^{-1}(O)$.

Proof. Let $(N, \Delta)$ be the lattice and fan associated to $X$. Let $U \subset X$ be the affine open subvariety containing the orbit $O$. Then the orbit $O$ corresponds to a simplicial cone $\sigma$ in $\Delta$. Let $N^{\prime}$ be the sublattice generated by the primitive elements of $\sigma$. Let $Y$ be the toric variety corresponding to $\left(N^{\prime}, \Delta\right)$ and $\mu$ be the natural finite dominant morphism corresponding to $\left(N^{\prime}, \Delta\right) \rightarrow(N, \Delta)$. By the construction of $\mu, \mu^{-1}(O)$ is smooth.

Proof of Main Lemma. Step 1. After $\mathbb{Q}$-factorization $q: X^{\prime} \rightarrow X$, we can assume that $X$ is a complete $\mathbb{Q}$-factorial toric variety [14] Corollary 3.6). Indeed, take points $P^{\prime}, Q^{\prime} \in X^{\prime}$ such that $q\left(P^{\prime}\right)=P$ and $q\left(Q^{\prime}\right)=Q$. The inverse image $S^{\prime}=q^{-1} S$ is a 
closed subvariety of codimension $\geq 2$, because $q$ is small. By Lemma 3.3, a weakly free rational curve $f^{\prime}: \mathbb{P}^{1} \rightarrow X^{\prime}$ over $\left\{P^{\prime}, Q^{\prime}\right\}$, disjoint from $S^{\prime} \backslash\left\{P^{\prime}, Q^{\prime}\right\}$ gives a weakly free rational curve $f=q \circ f^{\prime}: \mathbb{P}^{1} \rightarrow X$ over $\{P, Q\}$, disjoint from $S \backslash\{P, Q\}$.

Step 2. A weakly free rational curve can be moved from any smooth variety of codimension $\geq 2$ in the sense of Lemma 3.2. So we can reduce the proof of Main Lemma to the case $S=I(X)$, where $I(X)$ denotes the union of orbits of $X$ of codimension $\geq 2$. Since $X$ is a toric variety, Sing $X \subseteq I(X)$.

Indeed, for any subvariety $S \subseteq X$ of codimension $\geq 2$, suppose there is a sufficiently general weakly free rational curve $f: \mathbb{P}^{1} \rightarrow X$ over $P, Q \in X$, disjoint from $I(X) \backslash$ $\{P, Q\}$. Apply Lemma 3.2 to the subvariety $S$, and the weakly free rational curve $f$. Since Sing $X \subseteq I(X), s^{\prime}=0$ in Lemma 3.2, that is, $f\left(\mathbb{P}^{1}\right)$ is disjoint from $F_{1} \backslash\{P, Q\}, \ldots, F_{s} \backslash\{P, Q\}$. Then there exists a weakly free rational curve $f^{\prime}$, such that $f^{\prime}\left(\mathbb{P}^{1}\right)$ is disjoint from $\left((S \backslash\right.$ Sing $\left.X) \cup F_{1} \cup \cdots \cup F_{s}\right) \backslash\{P, Q\}=((S \backslash$ Sing $X) \cup$ Sing $X) \backslash\{P, Q\}=S \backslash\{P, Q\}$.

Step 3. Suppose that $I(X)$ consists of $\tilde{s}$ distinct orbits $O_{1}, \ldots, O_{\tilde{s}}$. Let $f: \mathbb{P}^{1} \rightarrow X$ be a sufficiently general weakly free rational curve over $P, Q$. By Lemma 3.1, we can assume that $f\left(\mathbb{P}^{1}\right)$ intersects with $O_{1} \backslash\{P, Q\}, \ldots, O_{s^{\prime}} \backslash\{P, Q\}$, and is disjoint from $O_{s^{\prime}+1} \backslash\{P, Q\}, \ldots, O_{\tilde{s}} \backslash\{P, Q\}$ for some $s^{\prime}$.

Notice that $s^{\prime}$ depends on the points $P, Q$ and the variety $X$. However, since $s^{\prime}$ is bounded by $\tilde{s}$, and $\tilde{s}$ is independent of the choice of $X$ in an isogeny class, there exists an $\bar{s}$ such that for any toric variety $Y$ in the isogeny class of $X$, and two distinct points $P^{\prime}, Q^{\prime} \in Y$, there exists a weakly free rational curve $f_{\bar{s}}^{\prime}: \mathbb{P}^{1} \rightarrow Y$ over $P^{\prime}, Q^{\prime}$, such that for any $1 \leq i \leq \tilde{s}$, if $f_{\bar{s}}^{\prime}\left(\mathbb{P}^{1}\right)$ intersects $O_{i}^{Y} \backslash\left\{P^{\prime}, Q^{\prime}\right\}$, then $1 \leq i \leq \bar{s}$, where $O_{i}^{Y}$ are orbits of $Y$ of codimension $\geq 2$. Furthermore, we can assume that $\operatorname{dim} O_{1}^{Y} \geq \operatorname{dim} O_{2}^{Y} \geq \cdots \geq \operatorname{dim} O_{s^{\prime}}^{Y} \geq \operatorname{dim} O_{s^{\prime}+1}^{Y} \geq \cdots \geq \operatorname{dim} O_{\tilde{s}}^{Y}$. This order is good for us, because $\cup_{j \geq s} O_{j}^{Y}$ is closed for any $s$.

We fix a complete toric variety $X$, two points $P, Q$ and a weakly free rational curve $f_{\bar{s}}$ over $P, Q$. By Lemmas 3.3 and 3.4, we can suppose that the orbit $O_{\bar{s}}$ is smooth. Indeed, by Lemma 3.4, there is an isogeny $\mu: Y \rightarrow X$ such that $O_{\bar{s}}^{Y}=\mu^{-1}\left(O_{\bar{s}}\right)$ is smooth. Let $P^{\prime}, Q^{\prime} \in Y$ such that $\mu\left(P^{\prime}\right)=P, \mu\left(Q^{\prime}\right)=Q$. Then the existence of a weakly free rational curve $f^{\prime}: \mathbb{P}^{1} \rightarrow Y$ over $P^{\prime}, Q^{\prime}$, disjoint from $O \bar{s} \cup \cdots \cup O_{\tilde{s}}^{Y}$, implies the existence of a weakly free rational curve $f: \mathbb{P}^{1} \rightarrow X$ over $P, Q$, disjoint from $O_{\bar{s}} \cup \cdots \cup O_{\tilde{s}}$, by Lemma 3.3 with $X^{\prime}=Y,\left\{P_{i}\right\}=\{P, Q\}$ and $S=O_{\bar{s}}^{Y} \cup O_{\bar{s}+1}^{Y} \cup \cdots \cup O_{\tilde{s}}^{Y}$.

Step 4. Now, we prove that there is a weakly free rational curve $f_{\bar{s}-1}$ over $P, Q$, such that for any $1 \leq i \leq \tilde{s}$, if $f_{\bar{s}-1}\left(\mathbb{P}^{1}\right)$ intersects $O_{i} \backslash\{P, Q\}$, then $1 \leq i \leq \bar{s}-1$. Indeed, we have the following two cases:

(1) If $f_{\bar{s}}\left(\mathbb{P}^{1}\right)$ is disjoint from $O_{\bar{s}} \backslash\{P, Q\}$, then let $f_{\bar{s}-1}=f_{\bar{s}}$.

(2) If $f_{\bar{s}}\left(\mathbb{P}^{1}\right)$ intersects $O_{\bar{s}} \backslash\{P, Q\}$, we apply Lemma 3.2 with $Z=O_{\bar{s}+1} \cup \cdots \cup$ $O_{\tilde{s}}$ and $S=O_{\bar{s}} \cup Z$. Notice that $S$ and $Z$ are closed subvarieties of $X$ of codimension $\geq 2$, and $O_{\bar{s}}$ is smooth. In particular, $S \backslash \operatorname{Sing} X \supseteq O_{\bar{s}}$. By assumption, $f_{\bar{s}}\left(\mathbb{P}^{1}\right) \cap(Z \backslash\{P, Q\})=\emptyset$. Therefore, by the Lemma, there exists a weakly free rational curve $f_{\bar{s}-1}$ on $X$, such that for any $1 \leq i \leq \tilde{s}$, if $f_{\bar{s}-1}\left(\mathbb{P}^{1}\right)$ intersects $O_{i} \backslash\{P, Q\}$, then $1 \leq i \leq \bar{s}-1$, and $f_{\bar{s}-1}\left(\mathbb{P}^{1}\right)$ is disjoint from $\left(O_{\bar{s}} \cup Z\right) \backslash\{P, Q\}=\left(O_{\bar{s}} \backslash\{P, Q\}\right) \cup\left(O_{\bar{s}+1} \backslash\{P, Q\}\right) \cup \cdots \cup\left(O_{\tilde{s}} \backslash\{P, Q\}\right)$.

Step 5 . By induction on $\bar{s}$, there is a weakly free rational curve $f_{0}$ over $P, Q$, disjoint from $I(X) \backslash\{P, Q\}$. 
Proof of Main Theorem. Step 1. First, let us consider $S=\operatorname{Sing} X$.

There is a free rational curve $f_{0}: C_{0} \cong \mathbb{P}^{1} \rightarrow X$ over $\{P, Q\}$, and it is disjoint from $\left\{P_{i}\right\} \cup S$ for $\operatorname{dim} X \geq 2$, where $P, Q \notin\left\{P_{i}\right\} \cup S$ are any two distinct smooth points on $X$. Indeed, we can apply Main Lemma to the subvariety $\left\{P_{i}\right\} \cup S$ and two smooth points $P, Q$. Since $f_{0}\left(\mathbb{P}^{1}\right)$ is in the smooth locus of $X, f_{0}$ is free over $\{P, Q\}$, and it is disjoint from $\left\{P_{i}\right\} \cup S$ for $\operatorname{dim} X \geq 2$. We can also suppose that the curve $f_{0}$ is birational onto its image (see [4] Theorem II.3.14).

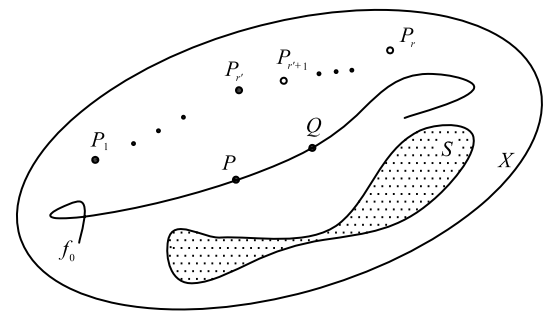

I. Assume that $P_{1}, \ldots, P_{r^{\prime}}$ are all the smooth points among $\left\{P_{i}\right\}$ of $X$, for some $r^{\prime}, 1 \leq r^{\prime} \leq r$. Since $f_{0}$ is birational onto its image, we can choose an isomorphism $\phi: \mathbb{P}^{1} \rightarrow C_{0}$, such that $P_{i}^{\prime}=f_{0}\left(\phi\left(p_{i}\right)\right)$ and $P_{i} \in X$ are all distinct. Thus, we identify $p_{i} \in \mathbb{P}^{1}$ with $\phi\left(p_{i}\right) \in C_{0}$ and $C_{0}=\mathbb{P}^{1}$ under the isomorphism. For each $j$, applying the Main Lemma to $S=\operatorname{Sing} X \cup\left\{P_{i}\right\}$ and points $P=P_{j}, Q=P_{j}^{\prime}$, there is a weakly free rational curve $f_{j}: C_{j} \cong \mathbb{P}^{1} \rightarrow X$ over $\left\{P_{j}, P_{j}^{\prime}\right\}$ with $f_{j}\left(0_{j}\right)=P_{j}$ and $f_{j}\left(\infty_{j}\right)=P_{j}^{\prime}$, where $0_{j}, \infty_{j} \in C_{j}$ for each $1 \leq j \leq r$, disjoint from $S \backslash\left\{P_{j}, P_{j}^{\prime}\right\}$.

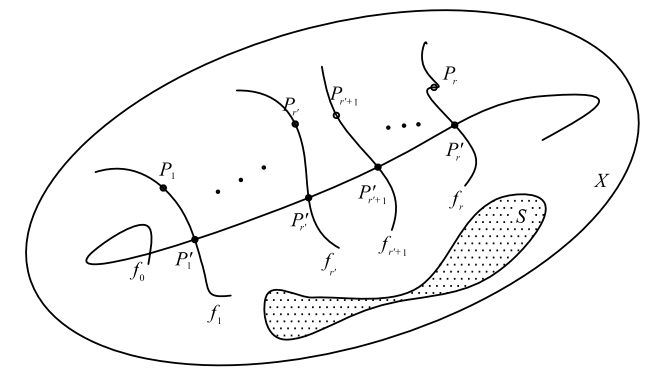

Applying the general statement of Theorem 2.2 to weakly free rational curves $f_{0}, f_{1}, \ldots, f_{r}$, and the set $\left\{P_{i}\right\}$ in Theorem 2.2 to the set $\left\{P_{r^{\prime}+1}, \ldots, P_{r}\right\}$ here, we get a resolution $\pi: X^{\prime} \rightarrow X$.

We construct a comb of smooth rational curves $C$ and a morphism $f: C \rightarrow X^{\prime}$ as follows.

For each $1 \leq i \leq r^{\prime}$, since $P_{i}$ and $P_{i}^{\prime}$ are smooth points, $f_{i}\left(\mathbb{P}^{1}\right)$ is contained in the smooth locus of $X$. Therefore $f_{i}$ is free for each $1 \leq i \leq r^{\prime}$ by [4] II.3.11. We identify the curve $f_{i}: C_{i} \cong \mathbb{P}^{1} \rightarrow X$ birationally with a free rational curve $f_{i}: C_{i} \cong \mathbb{P}^{1} \rightarrow X^{\prime}$. We also identify $P_{i} \in X$ with $P_{i} \in X^{\prime}$ for $1 \leq i \leq r^{\prime}$, and $P_{i}^{\prime} \in X$ with $P_{i}^{\prime} \in X^{\prime}$ for $1 \leq i \leq r$. More precisely, $f_{i}\left(0_{i}\right)=P_{i}$, where $0_{i} \in C_{i}, 1 \leq i \leq r^{\prime}$, and $f_{i}\left(\infty_{i}\right)=P_{i}^{\prime}$ where $\infty_{i} \in C_{i}, 1 \leq i \leq r$.

For each $r^{\prime}+1 \leq \bar{j} \leq r, P_{j}$ is singular. Let $f_{j}^{\prime}: C_{j} \cong \mathbb{P}^{1} \rightarrow X^{\prime}$ be the proper birational transformation of a sufficiently general deformation of $f_{j}$. Since $\pi: X^{\prime} \rightarrow X$ is a resolution in Theorem $2.2, f_{j}^{\prime}\left(C_{j}\right)$ intersects $\pi^{-1} P_{j}$ over $P_{j}$ only in divisorial points for $r^{\prime}+1 \leq j \leq r$, and is disjoint from the closure of $\pi^{-1}\left(S \backslash\left\{P_{i}\right\}\right)$. Let $Q_{j}$ be the 
point in $f_{j}^{\prime}\left(C_{j}\right) \cap \pi^{-1} P_{j}$ over $P_{j}$ for $r^{\prime}+1 \leq j \leq r$. We can suppose that $f_{i}$ is very free for $1 \leq i \leq r^{\prime}$ and $f_{j}^{\prime}$ is very free for $r^{\prime}+1 \leq j \leq r$ by [3] 1.1. or [4] II.3.11.

By the construction of $f_{i}, 1 \leq i \leq r^{\prime}$ and $f_{j}^{\prime}, r^{\prime}+1 \leq j \leq r, f_{i}\left(C_{i}\right)$ and $f_{j}^{\prime}\left(C_{j}\right)$ are disjoint from the closure of $\pi^{-1}\left(S \backslash\left\{P_{1}, \ldots, P_{r}\right\}\right)=\pi^{-1}\left(S \backslash\left\{P_{r^{\prime}+1}, \ldots, P_{r}\right\}\right)$.

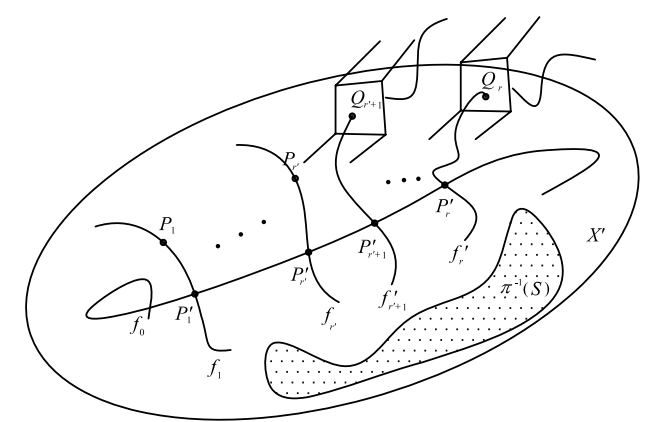

II. Gluing $\cup_{i=0}^{r} C_{i}$, we get a comb of smooth rational curves $C=\sum_{i=0}^{r} C_{i}$ and a morphism $f: C \rightarrow X^{\prime}$. Indeed, we identify points $\infty_{i} \in C_{i}$ with $p_{i} \in C_{0}$ for each $1 \leq i \leq r$. Then we have a comb of smooth rational curves $C=\sum_{i=0}^{r} C_{i}$ and a morphism $f: C \rightarrow X^{\prime}$ because $f_{0}\left(p_{i}\right)=f_{i}\left(\infty_{i}\right)=P_{i}^{\prime}$. Notice that $f(C)$ is disjoint from the closure of $\pi^{-1}\left(S \backslash\left\{P_{1}, \ldots, P_{r}\right\}\right)$.

In the end, $f: C \rightarrow X^{\prime}$ can be smoothed into a rational curve $f^{\prime}: \mathbb{P}^{1} \rightarrow X^{\prime}$ such that $f^{\prime}$ is free over $\left\{P_{i}\right\}, 1 \leq i \leq r^{\prime}$ and $\left\{Q_{j}\right\}, r^{\prime}+1 \leq j \leq r$ with $f^{\prime}\left(p_{i}\right)=P_{i}, 1 \leq i \leq r^{\prime}$ and $f^{\prime}\left(p_{j}\right)=Q_{j}, r^{\prime}+1 \leq j \leq r$, and is disjoint from the closure of $\pi^{-1}\left(S \backslash\left\{P_{1}, \ldots, \bar{P}_{r}\right\}\right)$ (See [4] II.7.6 ${ }^{1}$ ). By construction, for $\operatorname{dim} X \geq 2$ the smoothing $f^{\prime}$ is birational onto its image, and for $\operatorname{dim} X=1, f^{\prime}$ is finite onto its image.

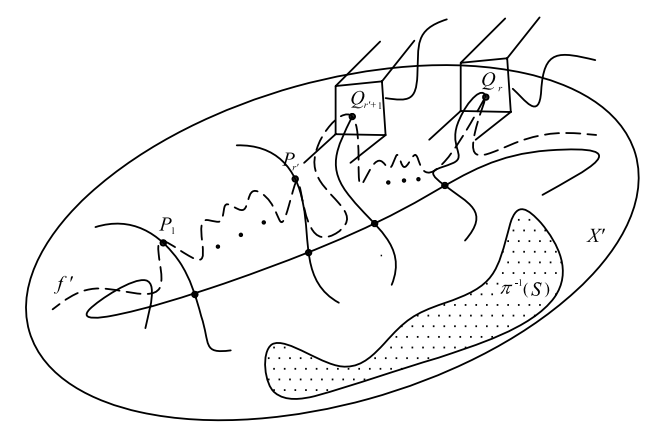

Step 2. Now we consider any closed subvariety $S$ of codimension $\geq 2$.

$\overline{\text { By Step }} 1$, there is a free rational curve $f^{\prime}: \mathbb{P}^{1} \rightarrow X^{\prime}$ over $\left\{P_{1}, \ldots, P_{r^{\prime}}, Q_{r^{\prime}+1}, \ldots\right.$, $\left.Q_{r}\right\}$, disjoint from the closure of $\pi^{-1}$ (Sing $\left.X \backslash\left\{P_{1}, \ldots, P_{r}\right\}\right)$, where $\pi: X^{\prime} \rightarrow X$ is the resolution in Step 1. On the other hand, $\pi^{-1}\left((S \backslash \operatorname{Sing} X) \backslash\left\{P_{1}, \ldots, P_{r}\right\}\right)$ is a codimension $\geq 2$ subvariety on $X^{\prime}$ by Theorem $\left.2.23^{\prime}\right)$. So a general deformation $f^{\prime \prime}$ of $f^{\prime}$ is free over $\left\{P_{1}, \ldots, P_{r^{\prime}}, Q_{r^{\prime}+1}, \ldots, Q_{r}\right\}$ with $f^{\prime \prime}\left(p_{i}\right)=P_{i}, 1 \leq i \leq r^{\prime}$ and $f^{\prime \prime}\left(p_{j}\right)=Q_{j}, r^{\prime}+1 \leq j \leq r$, disjoint from $\pi^{-1}\left((S \backslash \operatorname{Sing} X) \backslash\left\{P_{1}, \ldots, P_{r}\right\}\right)$ by $[4]$ II.3.7. Since $f^{\prime}$ is disjoint from the closure of $\pi^{-1}\left(\operatorname{Sing} X \backslash\left\{P_{1}, \ldots, P_{r}\right\}\right), f^{\prime \prime}$ is

\footnotetext{
${ }^{1}$ Theorem II.7.6 proves the existence of smoothing keeping points fixed. For combs, the same arguments of the proof gives more: a general smoothing of a comb is free over $B$, where $B$ is the set of intersection points of the handle and each teeth of the comb.
} 
disjoint from $\pi^{-1}\left(\operatorname{Sing} X \backslash\left\{P_{1}, \ldots, P_{r}\right\}\right)$. Hence $f^{\prime \prime}$ is disjoint from $\pi^{-1}$ (Sing $X \backslash$ $\left.\left\{P_{1}, \ldots, P_{r}\right\}\right) \cup \pi^{-1}\left((S \backslash \operatorname{Sing} X) \backslash\left\{P_{1}, \ldots, P_{r}\right\}\right)=\pi^{-1}\left(S \backslash\left\{P_{1}, \ldots, P_{r}\right\}\right)$. Therefore, $\pi f^{\prime \prime}$ is a general deformation of $\pi f^{\prime}$ over $\left\{P_{1}, \ldots, P_{r}\right\}$ with $\pi f^{\prime \prime}\left(p_{i}\right)=P_{i}, 1 \leq i \leq r$, disjoint from $S \backslash\left\{P_{1}, \ldots, P_{r}\right\}$, and thus $\pi f^{\prime}$ is a geometrically free rational curve over $\left\{P_{1}, \ldots, P_{r}\right\}$ on $X$ with $\pi f^{\prime}\left(p_{i}\right)=P_{i}, 1 \leq i \leq r$.

\section{Acknowledgments}

We are very grateful to Chenyang Xu and an anonymous referee for valuable comments and suggestions. Both authors were partially supported by NSF grant DMS-0701465.

\section{References}

[1] J. Kollár, Y. Miyaoka and S. Mori Rational connected varieties, J. Algebraic Geom. 1(3) (1992), 429-448.

[2] F. Campana, Connexité rationelle des variétés de fano, Ann. Sci. École Norm. Sup. 25(5) (1992), 539-545.

[3] J. Kollár, Y. Miyaoka and S. Mori, Rational connectedness and boundness of Fano manifolds, J. Differ. Geom. 36(3) (1992), 765-779.

[4] J. Kollár, Rational curves on algebraic varieties, Ergeb. Math. Grenz. 3 Folge, 32 . SpringerVerlag, Berlin, 1996.

[5] V. V. Shokurov, On rational connectedness, (Russian) Mat. Zametki 68(5) (2000), 771-782.

[6] Q. Zhang. Rational connectedness of log Q-Fano varieties, J. Reine Angew. Math. 590 (2006), $131-142$.

[7] C. Hacon and J. M Mernan, On Shokurov's rational connectedness conjecture, Duke Math. J. 138(1) (2007), 119-136.

[8] B. Hassett and Y. Tschinkel, Approximation at places of bad reduction for rationally connected varieties, Pure and Appl. Math. Q. 4(3) (2008), 743-766.

[9] S. Keel and J. Mckernan, Rational curves on quasi-projective surfaces, Mem. Amer. Math. Soc. 140(669) 1999, 153.

[10] C. Xu, Strong rational connectedness of surfaces, to appear in J. Reine Angew. Math.

[11] Yu. G. Prokhorov and V. V. Shokurov, Towards the second main theorem on complements, J. Algebraic Geom. 18(1) (2009), 151-199.

[12] W. Fulton, Introduction to toric varieties, Annals of Mathematics Studies, 131, Princeton University Press, 1993.

[13] T. Oda, Convex bodies and algebraic geometry, Springer-Verlag, 1988.

[14] O. Fujino, Notes on toric varieties from the Mori theoretic viewpoint, Tohoku Math. J. 55(4) (2003), 551-564.

[15] Y. Chen, Strong rational connectedness of toric varieties, PhD thesis, Johns Hopkins Univesity, 2009.

[16] H. Hironaka, Resolution of singularities of an algebraic variety over a field of characteristic zero. I, II, Ann. Math. 79(2) (1964), 109-203, 205-326.

[17] D. Abramovich and K. Karu, Weak semistable reduction in characteristic 0, Invent. Math. 139(2) (2000), 241-273.

Department of Mathematics, The Johns Hopkins University, Baltimore, MD 21218, USA Current address: Academy of Mathematics and System Science, Institute of Mathematics, Chinese Academy of Sciences, Beijing 100190, People's Republic of China

E-mail address: yifeichen@amss.ac.cn

Department of Mathematics, The Johns Hopkins University, Baltimore, MD 21218, USA

Steklov Mathematics Institute, Russian Academy of Sciences, Cubkina Str. 8, 119991, Moscow, Russia

E-mail address: shokurov@math.jhu.edu 
